a pity that, for example, Fig. 9.17, with its clear demonstration of load dropping off as cracks occur, does not come earlier in the book along with more discussion of such matters as unstable dynamic 'jumps' on cracking even in a deflection-controlled test, and uniqueness of numerical solution unless each individual crack is properly followed. On grounds of simplicity and adequate accuracy, Professor Chen currently advocates the 'smeared' approach to cracking, in which the material remains a continuum, but with an infinite number of infinitesimally narrow fissures over the relevant part of the finite element. One cannot help feeling that we need a finite element capable of coping with discrete cracks in any direction (without too many nodes), so that fracture mechanics can be properly applied in the analysis of reinforced concrete.

Be that as it may in the long-term future, Professor Chen's book is a splendid detailed survey of current non-linear constitutive laws. It will perhaps not be required reading for designers of ordinary concrete structures, but anyone interested in finite-element analysis should certainly have it on his shelf.

C. T. MORLEY

\title{
Creep and shrinkage in concrete structures
}

\author{
Editors: Z. P. Bazănt and F. H. Wittmann
}

Chichester, John Wiley \& Sons, 1982. $235 \times 160 \mathrm{~mm}$. Type-set, illustrated, hardback. pp. $x, 363$.

ISBN 0471104094 . Price: $\mathfrak{f 2 4 . 9 5 .}$

An International Symposium on Fundamental Research on Creep and Shrinkage of Concrete was held in September 1980 at the Swiss Federal Institute of Technology in Lausanne. In addition to a variety of papers of a purely research nature, ten invited lectures on selected topics were presented by authors drawn from Europe and North America. This volume consists of those invited contributions, some of which have been substantially revised and updated prior to publication.

The stated intention of the Symposium was, in the field of time-dependent deformations, to contribute to the closing of the gap between the typical materials science approach on the one hand and the methods developed by structural engineers on the other. To this end, the programme of the Symposium, and the invited contributions, were collected in three parts covering:

(1) the general material properties relating to the microstructure and macrostructure of cement paste and concrete;

(2) the measurement of creep and shrinkage, and mathematical modelling leading to constitutive equations;

(3) the analysis of engineering structures and the observation of the effects of time-dependent movements in such structures.

It is by no means easy to link such a wide range of interests and it is inevitable that the assembly of contributions from ten different authors can do nothing to improve the continuity of treatment. It follows that this book is not a text-book but rather a series of expositions with strong research connections, providing a number of individual views of topics throughout the intended range. Certainly, the structural engineer should not judge the contents from the title (and cover); he might well find that the coverage is both wider and more disjointed than he expected.

The first part of the book contains four chapters, one each for the contributions given by Professor J. F. Young (University of Illinois, USA), S. E. Pihlajavaara (Technical Research Centre of Finland), Professor J. W. Dougill (Imperial College, University of London) and E. Cinlar (Northwestern University, USA). The first two authors give overviews of the detailed structure of hardened cement paste and of the movement of moisture through the pores. The other two authors deal with probabilistic aspects of deformations and the mechanics of concrete as a two-phase material. Both go beyond the straight description of existing knowledge and include theoretical concepts of a more novel kind. Not too much is said in any of these chapters about the relevance of the topics to the reality of creep and shrinkage, and the value of this first part of the book lies in the extension of an understanding of the structure and behaviour of concrete. This may well provide the necessary foundation for further developments of a more practical kind.

The middle part of the book contains chapters written by C. D. Pomeroy (Cement and Concrete Association), Professor F. H. Wittmann (Swiss Federal Institute of Technology, Lausanne), and Professor Z. P. Bazănt (Northwestern University, USA). The contents cover a description of experimental methods for measuring creep and shrinkage, an exposition of the physical mechanisms that 
account for the time-dependent movements, and a very long discussion of mathematical models. The chapter on mechanisms provides a link with the first part of the book because most of the mechanisms relate to happenings at the microstructural level. The experimental chapter brings a welcome touch of practicality and makes an interesting contrast with the other two chapters of the middle part. The mathematical modelling is important in its demonstration of the relevant and powerful contribution made by the computer, especially in the ability to use numerical methods based on the integration of the effects taken over small time intervals. This is essentially a matter of considering rates of creep and shrinkage and could well be a pointer to future prediction methods based on rates of deformation rather than on the deformations themselves.

The third and last part of the book contains chapters written by C. A. Anderson (Los Alamos National Laboratory, USA), Professor W. H. Dilger (University of Calgary, Canada), and H. G. Russell (Portland Cement Association, USA), B. L. Meyers (Bechtel Power Corporation, USA) and M. A. Daye (Acres America Incorporated, USA). It is the most satisfactory part for the reader in that the coverage has the best balance. Thus the first author provides an example of sophistication in which computer-based constitutive laws are incorporated in a finite element analysis; this is complemented by the second author, who gives an overview of the various methods of time-dependent analysis available to the structural engineer with some elaboration, including examples of the author's preferred approach. The third chapter returns to reality by describing field observations on structures and by comparing observed and calculated deformations. It is sobering to find, at the end of a book largely concerned with theoretical matters, that existing analytical methods are still unable to produce more than approximate estimates of the behaviour of actual structures.

The reader will find that this volume contains many interesting facts and concepts on the creep and shrinkage of concrete, and on the analysis and measurement of the behaviour of concrete structures. These include ideas for the next generation of methods for estimating the magnitudes of creep and shrinkage, and demonstrations of the mechanics and techniques that can be readily adopted for computer processing. However, as indicated in the last paragraph of the last chapter 'the all inclusive empirical relationship or the complete theoretical formulation for the time-dependent deformation of concrete is as yet unobtainable'. This being so, a comprehensive discussion on the reliability and applicability of existing methods would have been appreciated, daunting though the preparation of such a comparison would be. In the end it may well be that future progress will hinge on the application of the methods described in the two most practical chapters - on experimentation and field observation.

\title{
Analysis of concrete box beams using small-computer capacity
}

\author{
B. I. Maisel
}

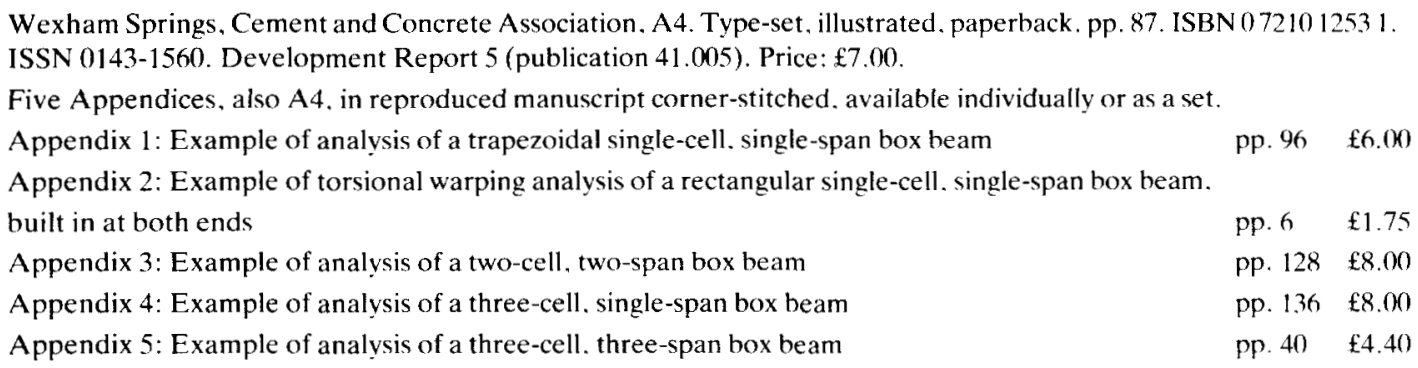

Appendices 1 to 5 as a set: $\mathbf{f 2 5 . 0 0}$

The second and final report of the Cement and Concrete Association on the analysis of box beams deals with prismatic multi-cell beams and consists of the main report and five Appendices totalling four hundred pages. It is clearly based on a very considerable amount of work of both theoretical and numeri- cal nature. The report consists of an extensive literature survey, a detailed explanation of Sedlacek's analytical treatment and the results of comparative studies of several box beam structures. The title of the report could be misleading, as 'small-computer capacity", when read in conjunction with the 